\title{
New Method for Accident Causation Analysis of Complex Socio-technical Systems
}

\author{
Jianwei Feng ${ }^{1, a}$, Song Wang 1, b \\ ${ }^{1}$ Equipment Engineering College, Engineering University of Armed Police Force of China, Xi'an \\ Shaanxi 710086, China. \\ aonesoon@163.com, ${ }^{b} 827754645 @ q q . c o m$
}

Keywords: Complex socio-technical systems, Accident causation, Risk emergence, Arena simulation.

\begin{abstract}
With the increasing structural complexity of complex socio-technical systems, which becomes also increasingly difficult to predict the catastrophic accident, the reason should be the existing accident causation theory incapable of modern complex systems accident analysis, spurned the system physical structure, based on perspective of large-scale systems theory, extracted accident causation factors from flaws of large systems, built the accident system model to simulate the cross-layer risk emergence by Arena software, the simulation results showed that the cross-layer risk emergence is a high-dimensional nonlinear growth process.
\end{abstract}

\section{Introduction}

Safety is the eternal topic of mankind, it has been accompanied by accidents of human development. With the development of productive forces, the degree of complexity of modern complex socio-technical system is also rising, and the complexity of many forms, and now the complexity of the system is gradually increased. System complexity increases, leading to a catastrophic accident after another ${ }^{[1,2,3]}$, such as: January 28, 1986 explosion of the United States, "Challenger" space shuttle, the April 1986 Chernobyl nuclear power plant tragedy, the explosion in March 2011 Fukushima nuclear power plant, August 12, 2015 in Tianjin, China dangerous goods warehouse explosion. Or mutations occur within the system architecture from these incidents (such as the "Challenger" space shuttle seal failure); or from the external environment mutation system (such as the southern ice storm in snow, cold and other sudden changes in weather caused electricity the collapse of the system). Environmental mutation by changing the system stability boundary, thus affecting the system component arrangement order, prompting the system's internal structure mutation, causing accidents, it is considered that the fundamental cause of accidents is that the internal structure of the mutant system, prevention of accidents countermeasure is to achieve an intrinsically safe system architecture.

Different systems have different systems of complex structure, complex systems from individual physical structure itself to study the correlation between structure and function is extremely difficult, and the relationship between structure and function of logic studied special complex systems, for the rest of the complex system is not applicable. In addition, for the accident, the cause of many systems are not only present in the interior of the physical system ${ }^{[4]}$, such as human error, environmental disturbances and other incidents belong to a complex system of objective external factors ${ }^{[5]}$. At this point you need to analyze complex systems abstracted as general issues accident to expand research, as can be under the premise of expanding the system boundary, according to certain rules to extract the mapping of complex systems in general accident factors, and access through a structured approach Factors progressive hierarchical model, namely to build a complex system that corresponds with the objective, and the greater the system boundary and the system has a strong element of accident system clustering properties. Further possible accidents through a variety of behavioral cognitive systems, particularly kinetic behavior of its risks, control measures to achieve security of complex systems. 


\section{Mapping from the physical structure of the accident structure}

Systems theory point out that the hierarchy system structure determines the system functions, complex systems and component interactions lead to the emergence of a specific function, and accident system composed by the accident factor also has a special hierarchy and complex component interactions accident factors interaction with the coupling between the accident led to the final ${ }^{[6]}$. To reveal a complex system of accident law, we need to jump out special research focus on the physical structure itself (easily lead to limitations in the reliability of the research area), but the system should pay more attention to the accident structure universality. Accidents cause mechanism based on structural analysis system drawn from the same accident can be used to guide a complex system of safety and accident prevention.

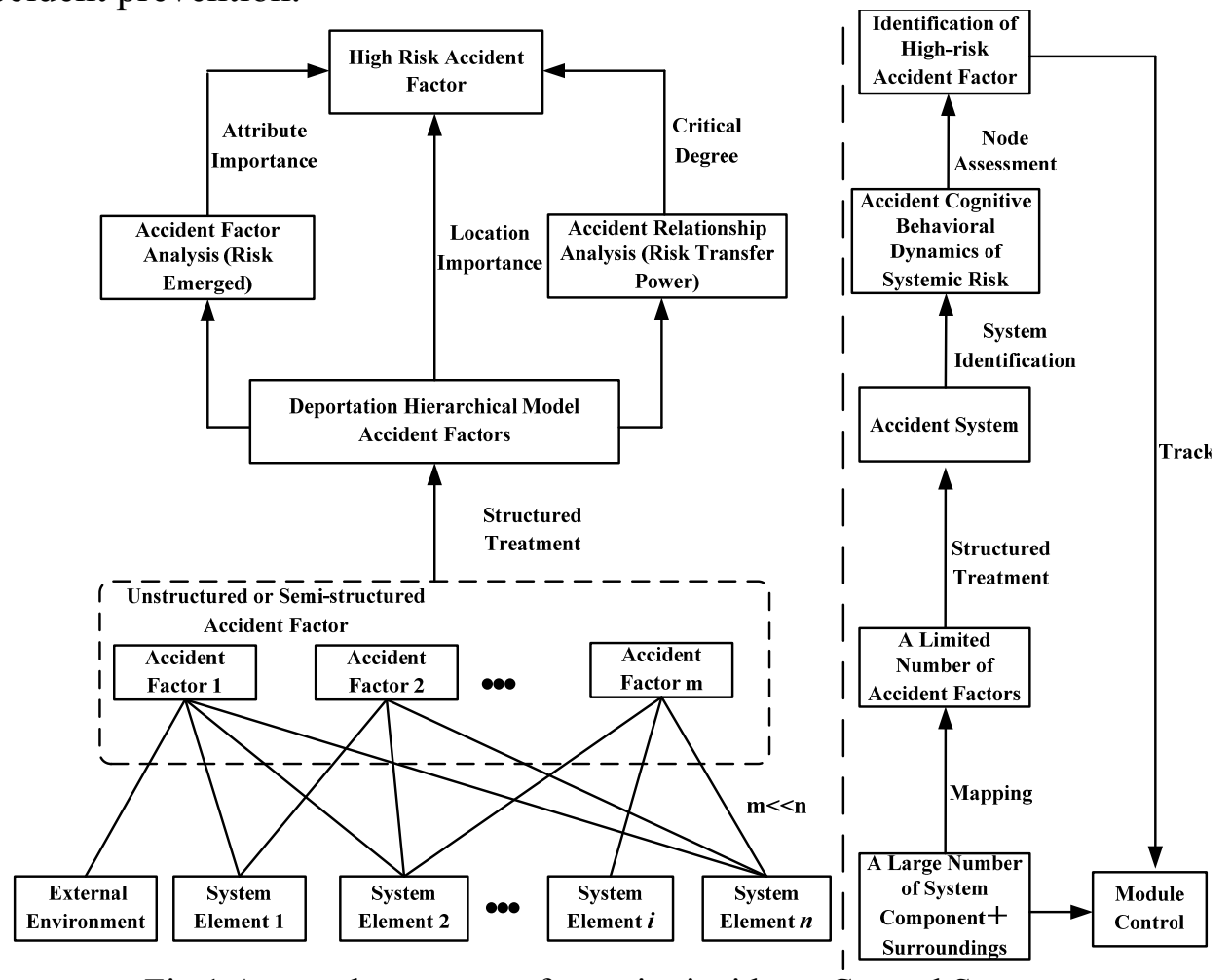

Fig 1 A complex system of security incidents Control Strategy

Accident system is a constituent element of the cause of accidents, which contains both the cause of cause of neighbors, but also contains the essence of causation. The figure shows the control strategy from a complex system of security incidents shown in Figure 1, the accident factor is an element having a high degree of clustering properties in the context of expanding the system boundary, the physical component obtained by class mapping. Thus, the accident factor is much less than the number of physical component, these factors often unstructured or semi-structured state presence. Through a structured approach, hierarchical structure model available accident factors, and then attribute importance by importance and relevance analysis, identification of high-risk factors of the accident, and then by tracing back to locate the failed physical component (also possible environmental disturbance or human error), its implementation module control, risk control within an acceptable range for the particular region.

\section{Arena emerging risk of cross-layer simulation}

References Security as a whole because of the emergence of a complex system security is the new property system level, the same as the antithesis of security incidents is the emergence of a holistic system, which is dangerous because a single point source is not necessarily trigger the final system accidents, safety and structure and function of the macroscale interaction inspired system on accidents are microscopic component ${ }^{[7]}$. 


\section{Simulation Model.}

In this section, project complex system, for example, consider the risk of the underlying technology (Technical Risk), the cost of risk (Cost Risk), risk management (Management Risk) and schedule risk (Schedule Risk) four underlying cause of the factors is how to lead a Development of research links (Node 5) the risk emerged, Arena simulation model is constructed as shown in Fig. The figure given by the four bottom node different risk distribution, technical risks assumed risk increase obey triangular distribution (TRIA $(0.5,0.75,1)$, the minimum is 0.5 , the most likely value of 0.75 and a maximum of 1 ), the cost of risk increased risk Poisson distribution (POIS (1), the average is 1), risk management increase the risk of a normal distribution (NORM (1,0.2), with mean 2 and variance 0.2) risk increased schedule risk obey an exponential distribution random (EXPO (1), the average is 1) to set the four biggest risk distribution is 100 . The simulation unit: hours (h).

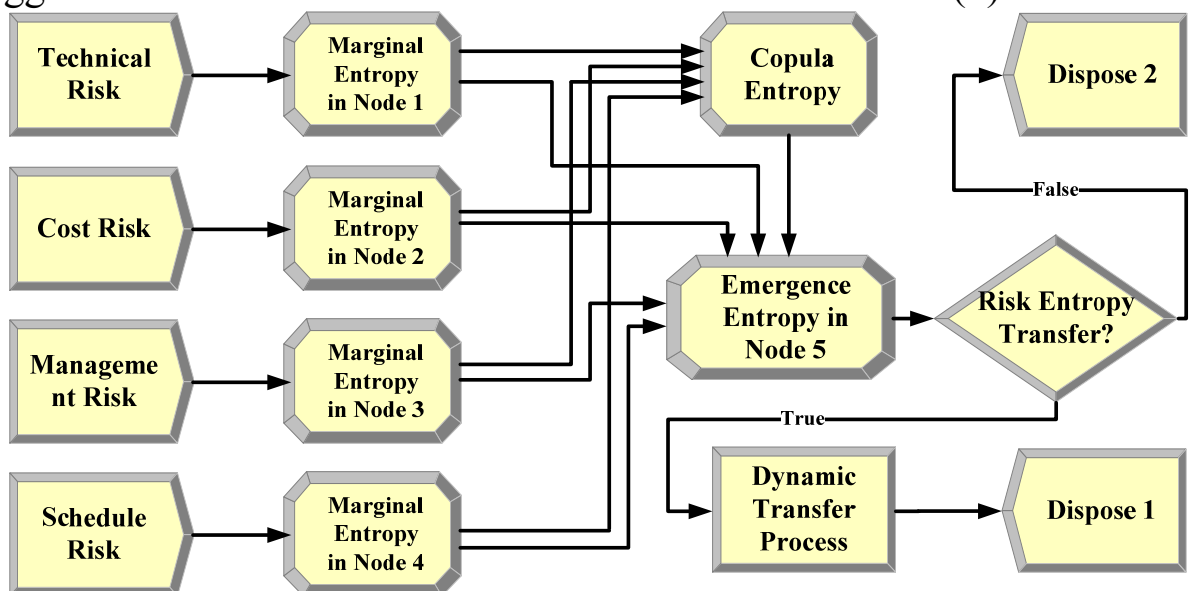

Fig 2 cross-layer model risk emerging Arena

By calculating the respective bottom node of an independent risk entropy (Marginal Entropy), take constant $\mathrm{K}=1$. By finding the four underlying distribution inverse functions, using the geometric mean and arithmetic mean assembly constructed Copula function to approximate Solving Coupled risk entropy (Copula Entropy), further calculates the value of cross-layer risk entropy (Emergence Entropy) is the resultant cross-layer node 5 (developed links) are emerging risk entropy.

\section{Simulation results and analysis.}

Get the bottom of the four risk factors through simulation independent risk entropy change curve shown in Figure 3, the figure shows, due to the different distribution, leading to the occurrence of entropy change curve risk difference, technical risk in the first triangular distribution reaches a maximum, normal second, followed by Poisson and exponential distribution. Since the initial set the same maximum risk of each underlying nodes, the maximum number of risks entropy achieved the same.
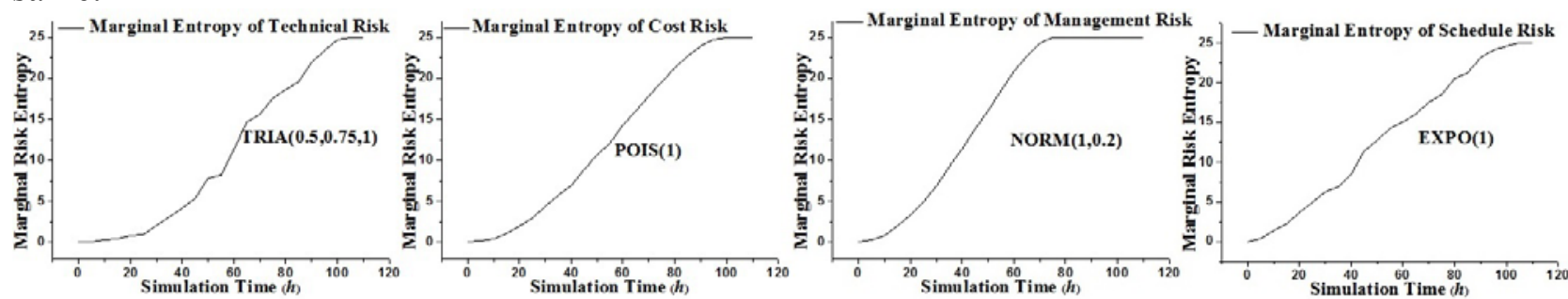

Fig 3 Bottom node independent risk entropy change curve

Figure 4 is an upper node Copula risk entropy and 5 (research aspects) risks resulting emergence of simulation graph from figure shows the trend of the two curves are essentially the same, but with a lower risk of risk management node entropy change of trend basically the same, but the probability of risk management risk increasing the density distribution of a Poisson distribution. Cross-layer node 5 of the emergence of risk entropy curve, not a simple linear growth, nor obey exponential growth curves, it can be non-linear polynomial fit. 


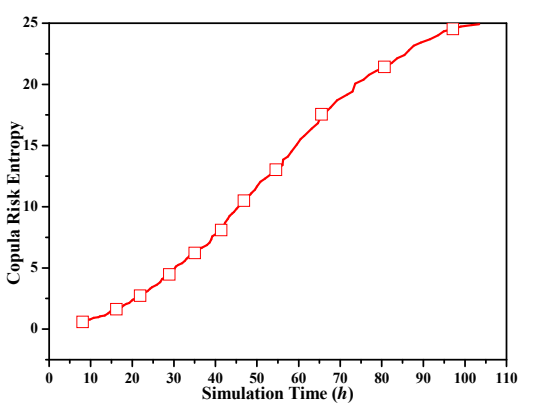

a. Copula Risk Entropy

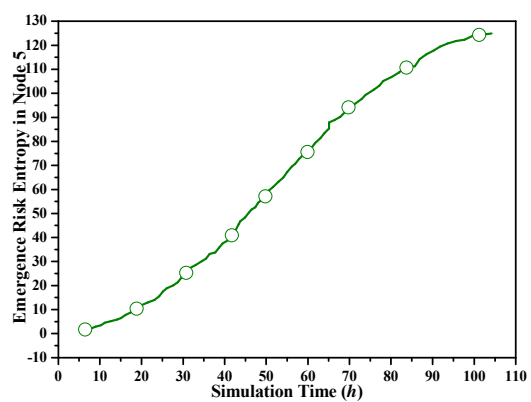

b. Emergence Risk Entropy in Node 5

Fig 4 Copula risk entropy and the emergence of the upper node risk curve

The risk of cross-layer respectively quadratic and cubic polynomial Fig. 4 (b) non-linear curve fitting, as shown in Figure 5 to give the emergence of a quadratic polynomial curve fitting and 6, as shown in the risk of the emergence of cross-layer three times order polynomial curve fitting. Both figures, a diagram is fitted curve and the original curve comparison chart, $b$ diagram is residual plots the fitting process.

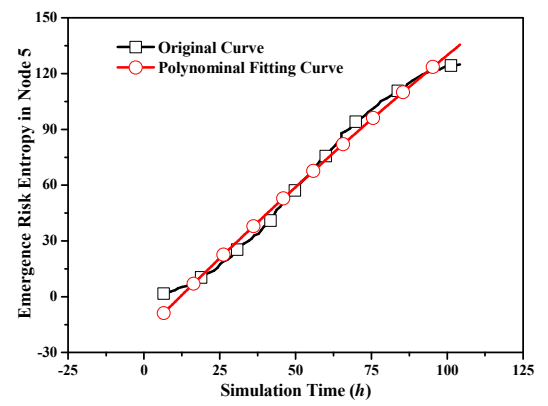

a. Curve fitting

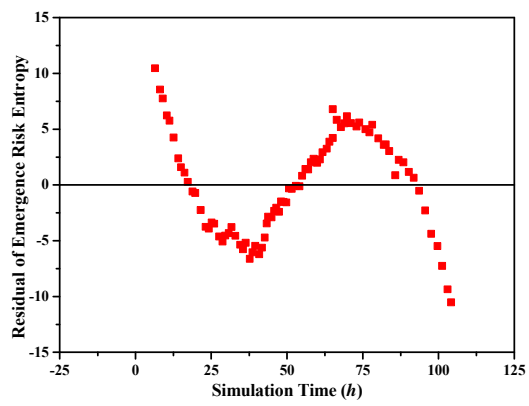

b. Residual plots

Fig 5 The risk of the emergence of cross-layer quadratic polynomial curve fitting

Figure 5, the simulation time $t$ as the independent variable, The emergence of risk entropy $H(t)$ as the dependent variable risk entropy, quadratic polynomial equation can be obtained as follows:

$$
H(t)=-0.00149 t^{2}+1.64 t-19.46
$$

Wherein the degree of curve fitting 0.9864 , although this number is very close to 1 (closer to 1 indicates that the better fit), but because of the quadratic term coefficient is small, so the curve approximates a linear distribution, entropy and the emergence of a real risk of discrepancies from the fitted curve can also be seen in FIG coincidence of the two curves is not high. And from the residual plot shows that the maximum deviation of the absolute value greater than 10, a little too large. This shows that the quadratic polynomial can not achieve better cross-layer nonlinear curve fitting risk emerged.

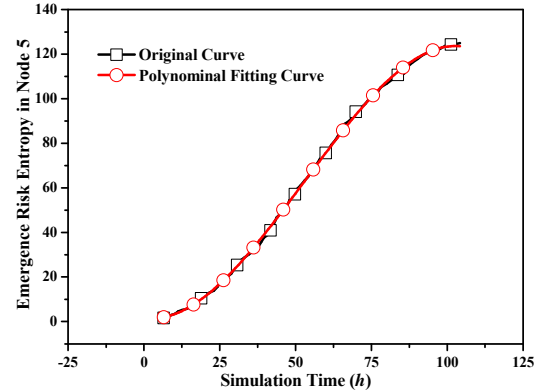

a. Curve fitting

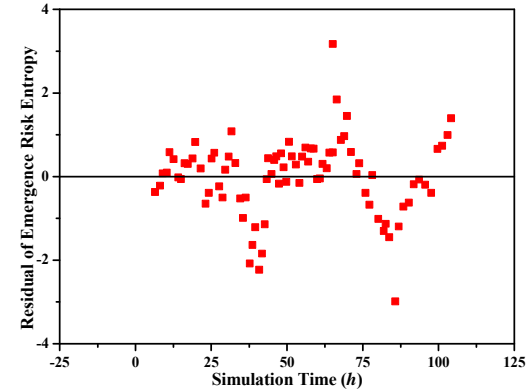

b. Residual plots

Fig 6 The risk of the emergence of cross-layer cubic polynomial curve fitting

Figure 6 is a cubic polynomial fitting result, the fitting equation is:

$$
H(t)=-2.453 t^{3}+0.039 t^{2}-0.212 t+1.801
$$

Wherein the degree of curve fitting 0.99945 significantly more optimized than the quadratic polynomial fitting results. And from the fitted curve shows that the original curve and polynomial curve fitting coincidence degree is very high, the maximum residual value is only 3 . This shows that the cubic polynomial can be used to fit nonlinear process risks emerge. It also shows that equation (2) 
can be used to calculate approximate entropy increase the risk of the emergence of cross-layer process.

Simulation and data fitting results show that the risk of the emergence of cross-layer growth process is a nonlinear high-dimensional, if time is the independent variable, which dynamic equations can be used as formula (2) cubic polynomial approximation is described. Such risk-based independent risk entropy and entropy Copula coupling, we can approximate measure dynamic processes emerging risks.

\section{Summary}

In order to explore the modern complex socio-technical systems accident causation analysis of new methods to establish the correspondence between the system structure and the hierarchy between the accident factor. Used Arena numerical analysis software underlying cause upper four nodes caused due cause of the node examples of risks emerge, and the use of polynomial fitting given the risk of cross-layer entropy emerged cubic polynomial equation, simulation and data fitting results show that the risk of the emergence of cross-layer growth process is a highly non-linear dimension.

\section{Acknowledgements}

This study was partially supported by the National Natural Science Foundation of China with the Grant number of 71401179.

\section{References}

[1] Allan J., McDonald. Lessons Learned but Forgotten from the Space Shuttle Challenger Accident[A]. Space 2004 Conference and Exhibit[C]. 28-30, September, 2004, San Diego, California, AIAA2004-5830: 1-11.

[2] Hayhurst, K. J. \& Holloway, C. M. Second Workshop on the Investigation and Reporting of Incidents and Accidents[EB/OL]. IRIA 2003, National Aeronautics and Space Administration Washington, DC 20546-0001, NASA/CP-2003-212642, Hampton, VA: NASA Langley Research Center. http://techreports.larc.nasa.gov/ltrs/ or http://ntrs.nasa.gov, 2003-1-1.

[3] Johnson, C. W. \& Holloway, C. M. A Longitudinal Analysis of the CausalFactors in Major Maritime Accidents in the USA and Canada(1996-2006)[A]. Proceedings of the 15th Safety-Critical Systems Symposium[C]. Bristol, UK, 13-15, February, Redmill, F. \& Anderson, T. (Eds.), The Safety of Systems, 2007, 85-94, Springer.

[4] A.L.C. Roelen, P.H. Lin, A.R. Hale. Accident models and organisational factors in air transport: The need for multi-method models [J]. Safety Science, 2011（49）: 5-10.

[5] Yan Fu Wang, Shahrzad Faghih Roohi, Xiu Ming Hu, et al. Investigations of Human and Organizational Factors in hazardous vapor accidents[J]. Journal of Hazardous Materials, 2011, 191 $(1-3): 69-82$.

[6] Qureshi Zahid H . A review of accident modelling approaches for complex critical Socio-technical systems[EB/OL] . http://www.dsto.defence.gov.au/corporate/reports/DSTOTR -2094.pdf, 2008-01-30.

[7] Tianyun Huang, Xue-bo Chen, Wei Wang, et al. Thinking in Emergence Control for Complex System[A]. Proceedings of the 8th World Congress on Intelligent Control and Automation[C]. June, Taipei, Taiwan, 2011: 21-25. 